\title{
EFEITOS DA COLHEITA MANUAL NA BIENALIDADE DO CAFEEIRO EM IJACI, MINAS GERAIS
}

\author{
Effects of manual harvesting on coffee (Coffea arabica L.) crop \\ biannuality in Ijaci, Minas Gerais
}

\author{
Fábio Moreira da Silva ${ }^{1}$, Marcelo de Carvalho Alves ${ }^{2}$, Júlio César Silva Souza ${ }^{3}$, Marcelo Silva de Oliveira ${ }^{4}$
}

\begin{abstract}
RESUMO
Analisou-se, neste trabalho, a relação entre a produtividade e a desfolha do cafeeiro durante o processo de colheita manual nas safras agrícolas de 2005, 2006 e 2007. O experimento foi desenvolvido na fazenda Cafua, localizada no município de Ijaci, sul de Minas Gerais, em uma área de 6,5ha de lavoura de cafeeiro (Coffea arabica L.) da variedade cultivada Catuaí Vermelho IAC-99. Foi demarcada na área uma malha irregular na distância de $25 \times 25$ e 50x50m, num total de 67 pontos amostrais, nos quais foram avaliados a produção de café (L.planta-1) obtida por meio da colheita no pano e a desfolha das plantas, quantificada com base no peso de folhas (kg. planta ${ }^{-1}$ ) após a colheita manual. Procedeu-se a análise de correlação de Pearson para verificar a relação linear entre a produtividade e a desfolha nos diferentes períodos avaliados. A dependência espacial dos dados também foi analisada por meio de ajustes de semivariogramas e interpolação por krigagem. Constatou-se que a colheita manual desfolhou mais em locais de maior produtividade, e apresentou redução da produtividade de café na lavoura no ano subsequente, pelo aumento da desfolha em plantas de maior produtividade e consequente redução da radiação fotossinteticamente ativa interceptada pelo dossel dessas plantas, ocasionando bienalidade da produção.
\end{abstract}

Termos para indexação: Agricultura de precisão, desfolha, produção, Coffea arabica.

\section{ABSTRACT}

This study analyzed the relation between coffee yield and defoliation during the process of manual harvest in the agricultural harvests of 2005, 2006 and 2007. The experiment was conducted at the Cafua farm, located in the municipality of Ijaci, Minas Gerais, in an area of 6.5 ha of coffee crop (Coffea arabica L.) Catuaí Vermelho IAC-99 cultivar. An irregular grid was demarcated in the area with a distance of $25 \times 25$ and $50 \times 50 \mathrm{~m}$, totaling 67 sampling points, which were evaluated coffee production $\left(\mathrm{L}_{\text {plant }}{ }^{-1}\right)$ obtained through harvest in cloth as well as defoliation of plants, quantified by the weight of leaves (A) $\left(\mathrm{kg} \mathrm{plant}^{-1}\right)$ after manual harvest. Pearson correlation analysis was used to verify the linear relationship between productivity and defoliation in different evaluated periods. The spatial dependence of the data was already analyzed through semivariogram adjustments and Kriging interpolation. Based on the analyses, it was found that manual harvest caused more defoliation in places with higher productivity and reduced the yield in the subsequent year, by the increase of defoliation on plants and reduction of the photosynthetically active radiation intercepted by the canopy of these plants, leading to the biannuality of the production.

Index terms: Precision agriculture, defoliation, production, Coffea arabica.

\section{(Recebido em 4 de julho de 2008 e aprovado 6 de outubro de 2009)}

\section{INTRODUÇÃO}

Com o processo de globalização da economia e a competitividade dos produtos agrícolas, passa a ser fundamental a adoção de novos métodos e técnicas de produção para o sucesso de qualquer atividade agrícola. Dentro desse contexto, surge a Agricultura de Precisão (Oliveira et al., 2007; Silva et al., 2008; Carvalho et al., 2009), como um conjunto de tecnologia capaz de auxiliar o produtor rural a identificar as estratégias a serem adotadas para aumentar a eficiência no gerenciamento da agricultura, maximizando a rentabilidade das colheitas, tornando o agronegócio mais competitivo.

Segundo Agrianual (2008), na safra 2007/2008, o Brasil foi o maior produtor mundial, seguido pelo Vietnã e a Colômbia. Apesar disso, a produtividade da cultura do café arábica (Coffea arabica L.) sofre oscilações, decorrentes de fatores climáticos (Carvalho et al., 2004), ocorrência de problemas fitossanitários (Chalfoun et al., 1978), característica fisiológica da cultura (Rena et al., 1996), sistema de plantio adotado, dentre outros fatores ainda não bem elucidados (Carvalho et al., 2006), acarretando na complexidade de previsão dessa variável e na redução do custo-benefício do produtor rural, pelo manejo da lavoura de forma homogênea.

Outro problema constatado nas regiões produtoras de café refere-se à variabilidade espacial e temporal da produtividade das plantas, sendo muito comum nas

1Universidade Federal de Lavras/UFLA - Departamento de Engenharia/DEG - Cx. P. 3037 - 37200-000 - Lavras, MG - famsilva@ufla.br

${ }^{2}$ Universidade Federal de Mato Grosso/UFMT - Departamento de Solos e Engenharia Rural - Cuiabá, MT

3Universidade Federal de Lavras/UFLA - Departamento de Engenharia/DEG - Lavras, MG

${ }^{4}$ Universidade Federal de Lavras/UFLA - Departamento de Ciências Exatas/DEX - Lavras, MG 
lavouras cafeeiras, plantas sem produção ao lado de plantas produtivas, bem como a bienalidade da produção, caracterizada pela variação de anos com alta e baixa produção (Carvalho et al., 2004).

Assim, no intuito de elucidar fatores que influenciam na oscilação espacial e temporal da produção de café, verificar a aplicação de uma técnica possível de ser utilizada para estudar esses fatores, bem como definir estratégias para o manejo sustentável da lavoura cafeeira, objetivouse caracterizar a estrutura, a magnitude de dependência espacial e mapear a variabilidade da produção de café e da desfolha das plantas em uma lavoura localizada em Ijaci, Minas Gerais.

\section{MATERIAL E MÉTODOS}

O experimento foi desenvolvido na fazenda Cafua, localizada no município de Ijací, sul de Minas Gerais, em uma área de 6,5 ha de lavoura de cafeeiro (Coffea arabica L.) da variedade cultivada Catuaí Vermelho IAC-99, com dezesseis anos de idade, no espaçamento de $3,8 \mathrm{~m}$ entre linhas e $1 \mathrm{~m}$ entre plantas, totalizando 2632 plantas.ha $^{-1}$. As coordenadas geográficas são de $21^{\circ} 10^{\prime} 11^{\prime \prime}$ de latitude sul e $44^{\circ} 58^{\prime} 37^{\prime \prime}$ de longitude oeste de Greenwich, com altitude média de $934 \mathrm{~m}$ e declividade de $0,84 \%$ no sentido nortesul e de $12 \%$, no sentido leste-oeste. Foi demarcada na área uma malha irregular na distância de 25x25 e 50x50m, num total de 67 pontos amostrais (Figura 1).

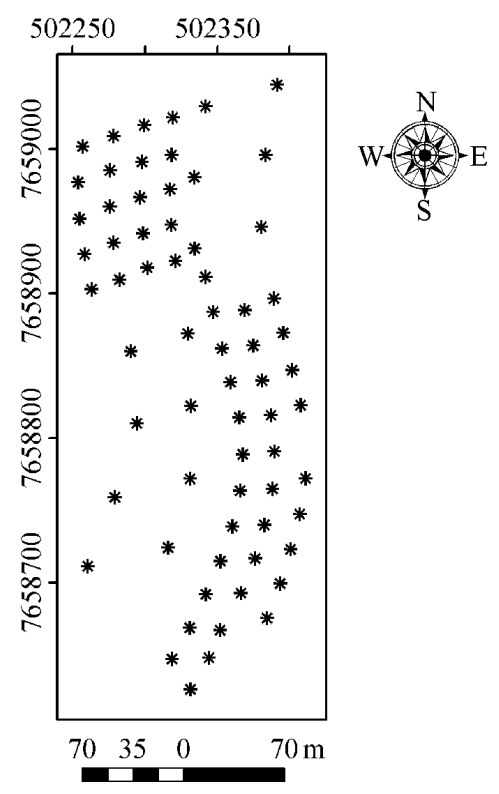

Figura 1 - Representação da malha amostral georreferenciada na área experimental em 2005, 2006 e 2007.
O georreferenciamento dos pontos foi realizado com GPS TRIMBLE 4600 LS $^{\circledR}$ e Estação Total Leica TC600 ${ }^{\circledR}$, com base em correção de cotas por coordenadas conhecidas no campus da Universidade Federal de Lavras.

Empregou-se o manejo convencional da área experimental durante o período avaliado. A adubação foi realizada em novembro de 2004, 2005 e 2006, por meio da aplicação de 42, 10 e $42 \mathrm{~kg} \cdot$ ha $^{-1}$ de N, P, K, via solo, respectivamente, seguida por pulverização de solução aquosa nas folhas com 0,24, 0,143 e 0,24 kg.ha-1 de Zn, B, KCL, respectivamente. Em janeiro de 2005, 2006 e 2007, aplicou-se 148, 37 e $148 \mathrm{~kg} \cdot \mathrm{ha}^{-1}$ de N, P, K, aplicados ao solo, respectivamente. Em fevereiro de 2005, 2006 e 2007, aplicou-se solução aquosa nas folhas com 0,086, 0,052 e 0,086 kg.ha-1 de Zn, B, KCL, respectivamente. Em setembro de 2006, aplicou-se $1700 \mathrm{~kg}$ ha $^{-1}$ de calcário no solo. O controle químico de insetos-praga na lavoura foi realizado em novembro de 2004, aplicando-se 0,6 L.ha-1 ${ }^{-1}$ Opus ${ }^{\circledR}$. Em abril de 2005, aplicou-se 0,01 kg.ha${ }^{1}$ de Amistar $^{\circledR}$ e 0,4 L.ha ${ }^{-1}$ de Opus ${ }^{\circledR}, 2,0$ L.ha $^{-1}$ de Endosulfan $\mathrm{AG}^{\circledast}$ e 2,0 L.ha ${ }^{-1}$ de Nimbus ${ }^{\circledast}$. Em novembro de 2005, aplicou-se 0,12 kg.ha-1 de Verdadero 600 $\mathrm{WG}^{\circledR}$. Em fevereiro de 2005 e 2006, aplicou-se 0,9 L.ha-1 de Nimbus $^{\circledR}, 1,4$ L.ha $^{-1}$ de Endosulfan $\mathrm{AG}^{\circledR}$ e 0,4 kg.ha ${ }^{-1}$ de Roundap $\mathrm{WG}^{\circledR}$. Em dezembro de 2006, aplicou-se 0,69 kg.ha ${ }^{-1}$ de Actara $250 W^{\circledR}, 0,045$ k.ha ${ }^{-1}$ de Amistar $^{\circledR}$ e 0,9 L.ha $^{-1}$ de Nimbus ${ }^{\circledR}$. Em fevereiro de 2007, aplicou-se 0,5 L.ha $^{-1}$ de Priori ${ }^{\circledR}$ e 1,4 L.ha-1 de Endosulfan AG $^{\circledR}$.

A produção de café (P) (L.planta ${ }^{-1}$ ) foi obtida por meio da colheita no pano e o volume coletado em um recipiente graduado. A desfolha foi quantificada com base no peso de folhas $(\mathrm{F})\left(\mathrm{kg}\right.$. planta $\left.\mathrm{pl}^{-1}\right)$ após a colheita manual. As amostras de produção e desfolha foram obtidas em 09/ 07/2005, 07/06/2006 e 11/07/2007.

Procedeu-se a análise de correlação de Pearson para verificar a relação linear entre a produtividade e a desfolha nos diferentes períodos avaliados. O coeficiente de correlação de Pearson foi calculado por (Webster \& Oliver, 2001).

A variabilidade e o padrão de dependência espacial da produção de café e da desfolha foram analisados por meio da geoestatística, baseado na metodologia de sistema de krigagem ordinária. As observações foram consideradas como realizações de uma função aleatória $Z(x)$, em que $x$ denota a localização espacial. A função aleatória satisfaz a pressuposição de estacionariedade da hipótese intrínseca, no domínio espacial de amostragem tal que (Olea,1999): $E[Z(x)]=m$ e $\operatorname{Var}[Z(x)-Z(x+h)]=2 \gamma(h)$, em que $V a r$ é a variância, $\gamma(h)$ é o semivariograma da função aleatória, $m$ é uma média constante e $h$ é uma distância vetorial no espaço amostral. 
Na geoestatística, a análise do modelo de semivariograma é uma etapa importante, visto que o modelo escolhido é a interpretação da estrutura de correlação espacial a ser utilizada nos procedimentos inferenciais da krigagem. O modelo relaciona a semivariância com o vetor distância (Burrough \& McDonnell, 1998).

Essa análise compreende o levantamento do semivariograma experimental e, posteriormente, o ajuste a uma família de modelos teóricos. É importante representar, no modelo ajustado, a tendência de $\hat{\gamma}(h)$ em relação a $h$, de forma que as estimativas baseadas na krigagem sejam mais exatas e confiáveis (Olea, 1999; Webster \& Oliver, 2001).

Os modelos de semivariograma teóricos isotrópicos esféricos foram ajustados por (Olea, 1999):

$$
\gamma(\mathrm{h})=\left\{\begin{array}{l}
\mathrm{C}\left(\frac{3}{2} \frac{\mathrm{h}}{\mathrm{A}}-\frac{1}{2}\left(\frac{\mathrm{h}}{\mathrm{A}}\right)^{3}\right), \quad 0 \leq \mathrm{h}<\mathrm{A} \\
\mathrm{C}, \mathrm{h} \geq \mathrm{A},
\end{array}\right.
$$

em que, $C$ é o patamar, A, é o alcance e $h$, a distância. $\mathrm{O}$ modelo esférico caracteriza-se por um comportamento linear próximo à origem e se torna achatado em maiores distâncias, atingindo o alcance quando $\mathrm{h}=\mathrm{A}$ (Isaaks \& Srivastava, 1989; Chilès \& Delfiner, 1999).

Os modelos foram escolhidos em função dos parâmetros dos semivariogramas, os coeficientes de determinação $\left(\mathrm{R}^{2}\right)$, a soma de quadrados do resíduo e a validação cruzada.

O método dos mínimos quadrados ponderado foi utilizado no ajuste dos modelos teóricos de semivariograma de acordo com a metodologia de Cressie (1985).

Após a análise variográfica, utilizou-se a interpolação por krigagem para mapear a variabilidade espacial da produção e da desfolha ao longo da área estudada. Na krigagem ordinária, a variação local da média é limitada pelo domínio de estacionariedade da média a uma vizinhança local $V(x)$ com o centro localizado na posição $x$ a ser estimada. O estimador linear é, portanto, uma combinação linear de $n$ variáveis aleatórias $Z\left(x_{i}\right)$ mais uma média local constante $m$ (Goovaerts, 1997):

$$
\hat{Z}(x)=\sum_{i=1}^{n} \lambda_{i} Z\left(x_{i}\right)+\left[1-\sum_{i=1}^{n} \lambda_{i}\right] m
$$

Nesse caso, a média local $m$ é filtrada do estimador linear $\hat{Z}\left(x_{i}\right)$ para que a soma dos pesos de krigagem $\lambda_{i}$ seja 1 . Assim, o estimador de krigagem ordinária é escrito como uma combinação linear de $n$ variáveis aleatórias $Z(x)$ :

$$
\hat{Z}(x)=\sum_{i=1}^{n} \lambda_{i} Z\left(x_{i}\right), \text { em que } \sum_{i=1}^{n} \lambda_{i}=1
$$

O erro esperado é $\mathrm{E}\left[\left\{\hat{\mathrm{Z}}\left(\mathrm{x}_{\mathrm{o}}\right)-\mathrm{Z}\left(\mathrm{x}_{\mathrm{o}}\right)\right\}^{2}\right]=0$ e a variância do estimador é (Webster \& Oliver, 2001):

$$
\begin{aligned}
\operatorname{var}\left[\hat{Z}\left(x_{o}\right)\right] & =E\left[\left\{\hat{Z}\left(x_{o}\right)-\hat{Z}\left(x_{o}\right)\right\}^{2}\right] \\
& =2 \sum_{i=1}^{n} \lambda_{i} \gamma\left(x_{i}, x_{o}\right)-\sum_{i=1}^{n} \sum_{j=1}^{n} \lambda_{i} \lambda_{j} \gamma\left(x_{i}, x_{j}\right)
\end{aligned}
$$

em que, $\gamma\left(\mathrm{x}_{\mathrm{i}}, \mathrm{x}_{\mathrm{j}}\right)$ é a semivariância entre o ponto amostral $i$ e os pontos $\mathrm{x}_{\mathrm{i}}$ e $\mathrm{x}_{\mathrm{j}}$ e, $\gamma\left(\mathrm{x}_{\mathrm{i}}, \mathrm{x}_{\mathrm{o}}\right)$, é a semivariância média entre o ponto amostral $i$ e o ponto $\mathrm{x}_{\mathrm{o}}$.

O próximo passo consiste em definir os pesos que minimizam essas variâncias, considerando-se a restrição que a soma dos pesos seja igual a 1. Para isso, utilizou-se o método de multiplicadores de Lagrange. Assim, definiuse uma função $f\left(\lambda_{i}, \phi\right)$ contendo a variância a ser minimizada e um termo adicional contendo o multiplicador de lagrange $\phi$ (Webster $\&$ Oliver, 2001):

$$
\left(\lambda_{i}, \phi\right)=\operatorname{var}\left[\hat{Z}\left(x_{o}\right)-Z\left(x_{o}\right)\right]-2 \phi\left\{\sum_{i=1}^{n} \lambda_{i}-1\right\}
$$

aplicando-se as derivadas parciais da função com relação à 0 os pesos do sistema de krigagem em blocos são obtidos por (Goovaerts, 1997):

$$
\left\{\begin{array}{l}
\sum_{\mathrm{i}=1}^{\mathrm{n}} \lambda_{\mathrm{i}} \gamma\left(\mathrm{x}_{\mathrm{i}}, \mathrm{x}_{\mathrm{j}}\right)+\phi\left(\mathrm{x}_{\mathrm{o}}\right)=\bar{\gamma}\left(\mathrm{x}_{\mathrm{j}}, \mathrm{x}_{\mathrm{o}}\right), \quad \forall \mathrm{j} \\
\sum_{\mathrm{i}=1}^{\mathrm{n}} \lambda_{\mathrm{i}}=1,
\end{array}\right.
$$

Em formato matricial, as equações da krigagem ordinária em blocos foram estimadas por (Webster \& Oliver, 2001):

$$
A \lambda=b \quad \approx \lambda=A^{-1} b
$$


em que, $A$ é a matriz de semivariância entre os pares de dados, $b$ é o vetor de semivariâncias entre cada par e a estimativa do bloco, $\lambda$ é o vetor de pesos e $\phi$ é o multiplicador de Lagrange:

$$
A=\left[\begin{array}{ccccc}
\gamma\left(x_{1}, x_{1}\right) & \gamma\left(x_{1}, x_{2}\right) & \ldots & \gamma\left(x_{1}, x_{n}\right) & 1 \\
\gamma\left(x_{2}, x_{1}\right) & \gamma\left(x_{2}, x_{2}\right) & \ldots & \gamma\left(x_{2}, x_{n}\right) & 1 \\
\cdot & \cdot & . & \cdot & \cdot \\
\cdot & \cdot & . & . & \cdot \\
\cdot & \cdot & . & . & \cdot \\
\gamma\left(x_{n}, x_{1}\right) & \gamma\left(x_{n}, x_{2}\right) & \ldots & \gamma\left(x_{n}, x_{n}\right) & 1 \\
1 & 1 & \ldots & 1 & 0
\end{array}\right],
$$

e a variância da krigagem é: $\sigma^{2}\left(x_{o}\right)=b^{T} \lambda$.

Preferiu-se a krigagem ordinária em blocos, em razão da estimativa por essa metodologia possibilitar obter superfície mais suavizada e menor variância da estimativa, quando comparada com a krigagem ordinária pontual (Burrough \& McDonnell, 1998). Com isso, foi possível representar a variabilidade espacial da infestação das pragas em função do espaçamento da cultura. O número de vizinhos mais próximos foi escolhido em função da validação cruzada.

A qualidade das estimativas obtidas pelo sistema de krigagem foi avaliada pela técnica da validação cruzada. A validação cruzada consiste em remover as observações e estimá-las pelo sistema de krigagem de forma a possibilitar comparar os valores observados e preditos para se determinar a acurácia e a precisão da krigagem (Isaaks \& Srivastava, 1989; Cressie, 1993; Goovaerts, 1997; Chilès \& Delfiner, 1999). Na validação da krigagem, o erro reduzido da krigagem foi definido por:

$$
E R=\frac{\sum_{i=1}^{n}\left(\hat{Z}\left(x_{i}\right)-Z\left(x_{i}\right)\right) / \hat{\sigma}\left(x_{i}\right)}{n} \text {, e a variância do erro }
$$

reduzido foi definida por:

$$
\mathrm{VER}=\sqrt{\frac{\sum_{\mathrm{i}=1}^{\mathrm{n}}\left[\left(\hat{\mathrm{Z}}\left(\mathrm{x}_{\mathrm{i}}\right)-\mathrm{Z}\left(\mathrm{x}_{\mathrm{i}}\right)\right) / \hat{\sigma}\left(\mathrm{x}_{\mathrm{i}}\right)\right]^{2}}{\mathrm{n}}},
$$

em que $\hat{Z}\left(x_{i}\right)$ é o valor estimado pelo sistema de krigagem, $z\left(x_{i}\right)$ é o valor observado e $\hat{\sigma}\left(x_{i}\right)$ o erro padrão da predição para o local $x_{i}$. Sob condições ideais, o erro reduzido da krigagem deve ser aproximadamente 0 e a variância do erro reduzido da estimativa deve ser aproximadamente 1 (Cressie, 1993).$$
\lambda=\left[\begin{array}{c}
\lambda_{1} \\
\lambda_{2} \\
\cdot \\
\cdot \\
\lambda_{n} \\
\phi
\end{array}\right]
$$$$
\mathrm{b}=\left[\begin{array}{c}
\gamma\left(\mathrm{x}_{1}, \mathrm{x}_{\mathrm{o}}\right) \\
\gamma\left(\mathrm{x}_{2}, \mathrm{x}_{\mathrm{o}}\right) \\
\cdot \\
\cdot \\
\gamma\left(\mathrm{x}_{\mathrm{n}}, \mathrm{x}_{\mathrm{o}}\right) \\
1
\end{array}\right]
$$

\section{RESULTADOS E DISCUSSÃO}

Com base na correlação de Pearson, observou-se correlação linear positiva significativa $(\mathrm{p} \leq 0,001)$ entre a produtividade e a desfolha avaliadas na mesma safra agrícola, sendo de 0,86 para o ano de 2005, de 0,91 para 2006 e de 0,89 para 2007, bem como correlação linear negativa significativa $(\mathrm{p} \leq 0,001)$ da produção da safra de 2005 com a de $2006(r=-0,70)$ e da produção da safra de 2006 com a de 2007 ( $\mathrm{r}=-0,52)$, caracterizando-se a bienalidade da produção (Tabela 1).

Com base na análise geoestatística, foi possível constatar a dependência espacial da produção de café e da desfolha causada pela colheita manual na lavoura nas safras de 2005, 2006 e 2007, pois o valor absoluto da diferença entre duas amostras observadas cresceu com o aumento da distância entre elas, até um valor no qual os efeitos locais não apresentaram mais influência, culminando na estabilidade do semivariograma experimental a partir da distância que separa o variabilidade estruturada da aleatória (Figura 2).

Foram ajustados modelos de semivariogramas esféricos ao semivariograma experimental, tanto para os dados de produção como para os dados de desfolha. Nesse caso, observaram valores semelhantes do alcance dos modelos utilizados para caracterizar a estrutura de dependência espacial da produção e da desfolha em 2005 , com magnitude $21,3 \mathrm{~m}$, em 2006, com magnitude de 27,6 e 34m, bem com em 2007, com magnitude de 36,0 e 36,2m (Figura 2). Faulin et al. (2004), ao estudar a variabilidade espacial da produção de café por meio de sensor adaptado a uma colhedora Jacto modelo K-3, em um talhão de 8,3ha da cultivar Mundo Novo, em Gália, São Paulo, observou, com base em semivariograma esférico ajustado aos dados de produção de café, alcance de $29,6 \mathrm{~m}$, próximo aos valores obtidos no presente estudo. 
Tabela 1 - Matriz de correlação entre as observações de produtividade (P) e de desfolha (D) em 09/07/2005, 07/06/2006, 11/07/2007.

\begin{tabular}{lccccc}
\hline & P 2007 & P 2006 & P 2005 & D 2007 & D 2006 \\
\hline P 07/06/2006 & $-0,52^{*}$ & & & \\
P 09/07/2005 & $0,39^{*}$ & $-0,70^{*}$ & & \\
D 11/07/2007 & $0,89^{*}$ & $-0,58^{*}$ & $0,49^{*}$ & $-0,56^{*}$ & \\
D 07/06/2006 & $-0,58^{*}$ & $0,91^{*}$ & $-0,67^{*}$ & $0,49^{*}$ & $-0,56^{*}$ \\
D 09/07/2005 & $0,42^{*}$ & $-0,62^{*}$ & $0,86^{*}$ & & \\
\hline
\end{tabular}

*p-valor significativo a $1 \%$, pelo teste $\mathrm{t}$
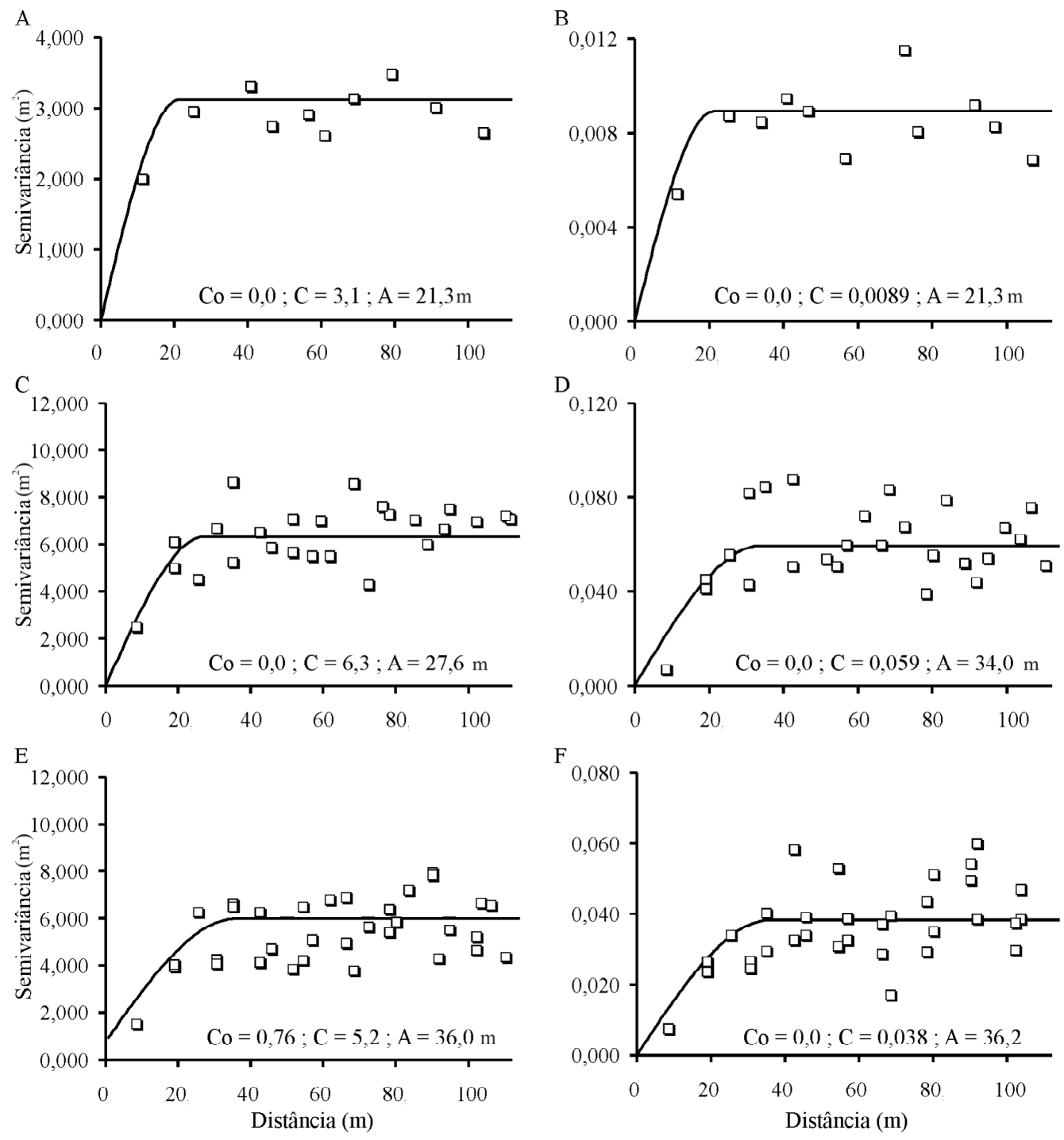

Figura 2 - Coeficientes e parâmetros de semivariogramas esféricos isotrópicos (-) ajustados aos semivariogramas esperimentais $(\square)$ referentes às observações de produtividade (A, C, E) e de desfolha (B, D, F) em 09/07/2005, 07/06/ 2006 e 11/07/2007. Co = efeito pepita, $\mathrm{C}=$ patamar e $\mathrm{A}=$ alcance. 
Posteriormente, por meio do método da krigagem, estimaram-se valores de produção e desfolha na lavoura com base na estrutura de dependência espacial dos modelos de semivariogramas esféricos. Observou-se bom desempenho da krigagem para estimar a produção e a desfolha na lavoura em todos os períodos avaliados, pois o erro padrão médio reduzido da estimativa foi, aproximadamente, zero e o quadrado médio do erro padrão reduzido foi aproximadamente um, sendo condizente com as condições ideais de um estimador espacial conforme descrito por Cressie (1993) (Figura 3).
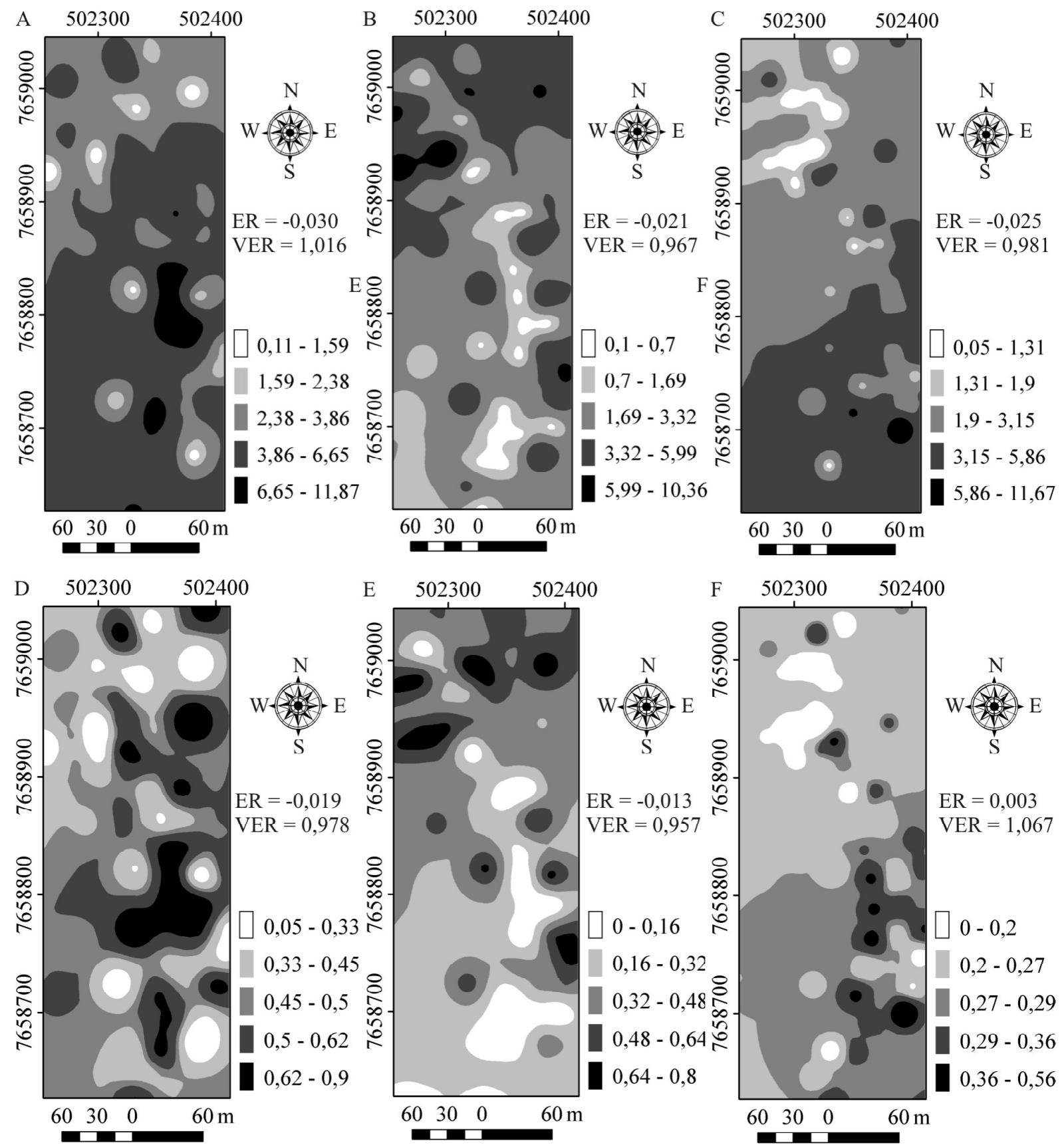

Figura 3 - Mapas da produtividade (A, B, C) e da desfolha (D, E, F) em 09/07/2005, 07/06/2006 e 11/07/2007, com os respectivos coeficientes de erro reduzido (ER) e variância do erro reduzido (VER) das estimativas. 
Observando-se os mapas de krigagem, constatou-se correspondência direta da variabilidade espacial de plantas com maior produtividade e maior desfolha no mesmo ano, bem como correspondência inversa em anos distintos, caracterizando a bienalidade da produtividade da cultura do café ao longo do espaço e do tempo. Os mapas de produção apresentaram valores variando de 0 a 11,8 L.planta ${ }^{-1}$ em 2005, de 0 a 10,3 L.planta ${ }^{-1}$ em 2006 e de de 0 a 11,8 L.planta ${ }^{-1}$ em 2007, os quais se encontram próximos dos observados nos estudos realizados por Balastreire (2001) e Silva et al. (2007). A desfolha das plantas causada pela colheita manual apresentou valores de 0,05 a 0,9 kg.planta ${ }^{-1}$ em 2005, 0 a 0,8 kg.planta ${ }^{-1}$ em 2006 e 0 a 0,56 kg.planta ${ }^{-1}$ em 2007. Os valores de desfolha observados no presente estudo foram próximos do encontrado por Silva et al. (2000), situado próximo a 0,640 kg.planta ${ }^{-1}$ (Figura 3).

Segundo Bártholo \& Guimarães (1997), um dos principais danos causados ao cafeeiro pela ação da colheita é a desfolha. Segundo os autores, a ocorrência frequente de tal fato proporcionará estresse da planta e redução da sua longevidade. Com a desfolha, a planta produzirá menos no ano seguinte, uma vez que utilizará suas reservas para a recomposição da vegetação e, por conseguinte, terá uma menor frutificação. Assim, de acordo com Rena et al. (1996), de forma geral, as plantas muito produtivas ficam debilitadas em função do dreno de nutrientes das folhas para os frutos em anos de alta produtividade, pois o cafeeiro não regula a carga de frutos, provocando distúrbios fisiológicos na planta, culminando em queda de folhas, redução da fotossíntese e baixa produtividade no ano subseqüente.

Portanto, com base nos resultados obtidos, sob as condições avaliadas na área experimental, a colheita manual foi um dos principais fatores que determinou a redução da produtividade de café na lavoura no ano subsequente, em função do dano físico causado pela desfolha na derriça dos frutos de café nas plantas, causando redução da radiação fotossinteticamente ativa interceptada pelo dossel e alterações fisiológicas no metabolismo dessas plantas.

\section{CONCLUSÕES}

Foi possível caracterizar a estrutura, a magnitude de dependência espacial e mapear a variabilidade da produção de café e da desfolhas das plantas em uma lavoura localizada em Ijaci, Minas Gerais.

Observou-se relação entre a produtividade e a desfolha do cafeeiro após a colheita manual por metodologia de análise estatística e geoestatística.

\section{REFERÊNCIAS BIBLIOGRÁFICAS}

AGRIANUAL. Anuário da agricultura brasileira. São Paulo: FNP, 2008. 504p.

BALASTREIRE, L.A. Agricultura de precisão: mapeamento da produtividade de uma cultura de café. In: CONGRESSO BRASILEIRO DE ENGENHARIA AGRÍCOLA, 30., 2001, Foz do Iguaçu. Anais... Jaboticabal: SBEA, 2001. CD-ROM.

BÁRTHOLO, G.F.; GUIMARÃES, P.T.G. Cuidados na colheita e preparo do café. Informe Agropecuário, Belo Horizonte, v.19, n.187, p.33-42, 1997.

\section{BURROUGH, P.A.; McDONNELL, R.A. Principles of} geographical information systems: spatial information systems and geostatistics. 2.ed. Oxford: Oxford University, 1998. 333p.

CARVALHO, G. R.; BOTELHO, C. E.; BARTHOLO, G. F.; PEREIRA, A. A.; NOGUEIRA, Â. M.; CARVALHO, A. M. de. comportamento de progenies $\bar{F}_{4}$ obtidas por cruzamentos de 'Icatu' com 'Catimor'. Ciência e Agrotecnologia, Lavras, v.33, n.1, p.47-52, jan./fev., 2009.

CARVALHO, G.R.; MENDES, A.N.G.; BARTHOLO, G.F.; NOGUEIRA, Â.M.; AMARAL, M.A. Avaliação de produtividade de progênies de cafeeiro em dois sistemas de plantio. Ciência \& Agrotecnologia, Lavras, v.30, n.5, p.838-843, 2006.

CARVALHO, L.G.; SEDIYAMA, G.C.; CECON, P.R.; ALVES, H.M.R. A regression model to predict coffee productivity in Southern Minas Gerais, Brazil. Revista Brasileira de Engenharia Agrícola e Ambiental, Campina Grande, v.8, n.2/3, p.204-211, 2004.

CHALFOUN, S.M.; SILVA, C.M.; PEREIRA, A.A.; PAIVA, F.A. Relação entre diferentes níveis de infecção de ferrugem (Hemileia vastatrix Berk. \& Br.) e produção dos cafeeiros (Coffea arabica L.) em algumas localidades de Minas Gerais. In: CONGRESSO BRASILEIRO DE PESQUISAS CAFEEIRAS, 6., 1978, Ribeirão Preto. Resumos... Rio de Janeiro, 1978. p.392394.

CHILÈS, J.P.; DELFINER, P. Geostatistics: modeling spatial uncertainty. New York: J.Wiley, 1999. 695p. 
CRESSIE, N. Statistics for spatial data. New York: J.Wiley, 1993. 900p.

CRESSIE, N. Fitting variogram models by weighted least squares. International Association for Mathematical Geology, v.17, p.653-702, 1985.

FAULIN, G.D.C.; MOLIN, J.P.; MAGALHÃES, R.P.; STANISLAVSKI, W.M. Estudo da espacialidade da maturação de café (Coffea arabica L.) visando a confecção de mapas de produtividade. In: CONGRESSO BRASILEIRO DE AGRICULTURADE PRECISÃO CONBAP, 2004, Piracicaba. Anais... Piracicaba, 2004. CDROM.

GOOVAERTS, P. Geostatistics for natural resources evaluation. New York: Oxford University, 1997. 483p.

ISAAKS, E.H.; SRIVASTAVA, R.M. Applied geostatistics. New York: Oxford University, 1989. 561p.

OLEA, R.A. Geostatistics for engineers and earth scientists. Norwell: Kluwer Academic, 1999. 324p.

OLIVEIRA, E. de; SILVA, F. M. da; GUIMARÃES, R. J; SOUZA, Z. M. de. Eliminação de linhas em cafeeiros adensados por meio semimecanizado. Ciência e
Agrotecnologia, Lavras, v.31, n.6, p.1826-1830, nov./dez., 2007.

RENA, A.B.; NACIF, A.P.; GONTIJO, P.T.; PEREIRA, A.A. Fisiologia do cafeeiro em plantios adensados. In: SIMPÓSIO INTERNACIONAL SOBRE CAFÉ ADENSADO, 1994, Londrina. Anais... Londrina: Instituto Agronômico do Paraná, 1996. p.73-85.

SILVA, F.M.; RODRIGUES, R.F.; SALVADOR, N.; TOURINO, E.S.; SILVA, S.S.S. Custo da colheita mecanizada de café com colhedoras automotrizes no Sul de Minas. Engenharia na Agricultura, Viçosa, v.8, n.1, p.54-60, 2000.

SILVA, F. M. da; SOUZA, Z. M. de; FIGUEIREDO, C. A. P. de; VIEIRA, L. H. de S.; OLIVEIRA, E. de. Variabilidade espacial de atributos químicos e produtividade da cultura do café em duas safras agrícolas. Ciência e Agrotecnologia, Lavras, v.32, n.1, p.231-241, jan./fev., 2008.

SILVA, F.M.; SOUZA, Z.M.; FIGUEIREDO, C.A.P.; MACHADO, R.V. Variabilidade espacial de atributos químicos e de produtividade na cultura do café. Ciência Rural, Santa Maria, v.37, n.2, p.401-407, 2007.

WEBSTER, R.; OLIVER, M. Geostatistics for environmental scientists. London: J.Wiley, 2001. 271p. 